\title{
Holoprosencefalia Semilobar y Malformaciones Asociadas: Reporte de Caso y Algunas Consideraciones
}

\author{
Semilobar Holoprosencephaly and Associated Malformations: Case Report and Some Considerations
}

Hugo Alberto Cervantes-Flores ${ }^{1}$; Samuel Bencomo-Loeppky ${ }^{1}$; Jorge Arturo García-Rizk ${ }^{1}$; Margarita Levario-Carrillo² ${ }^{2}$ Sandra Alicia Reza-López ${ }^{2}$; Martha Elena Loya-Loya ${ }^{3}$; Luz Helena Sanín ${ }^{3}$; Ricardo Fierro-Murga ${ }^{4}$ \& Dora Virginia Chávez-Corral²;

CERVANTES-FLORES, H. A.; BENCOMO-LOEPPKY, S.; GARCÍA-RIZK, J. A.; LEVARIO-CARRILLO, M.; REZALÓPEZ, S.A.; LOYA-LOYA, M. E.; SANÍN, L. H.; FIERRO-MURGA, R. \& CHÁVEZ-CORRAL, D. V. Holoprosencefalia semilobar y malformaciones asociadas: Reporte de caso y algunas consideraciones. Int. J. Morphol., 37(1):123-127, 2019.

RESUMEN: El defecto más común del prosencéfalo es la holoprosencefalia (HPE), caracterizada por ausencia en la división del prosencéfalo. La holoprosencefalia tiene una prevalencia de 1/10.000 en recién nacidos; la ciclopía de 1/100.000 nacidos y la agnatia asociada a holoprosencefalia de 0,8 a $10 \%$. El objetivo fue describir las características morfológicas e histopatológicas de un feto humano con holoprosencefalia y sus malformaciones asociadas. Se estudió un feto masculino. Se le realizó microdisección bajo el estereomicroscopio, toma de microfotografías con cámara AxioCam y software AxioVision 4.8, y estudio histopatológico. La edad gestacional estimada fue de 12,4-13,2 semanas, encontrándose como hallazgos la HPE semilobar asociada a ciclopía, esbozo oral hipoplásico sin apertura oral, cubierta por una membrana y ausencia de labios. El estudio histopatológico reportó: ojo con lente, retina y córnea únicos; en la cara, probóscide con cartílago tubular en formación asociado a mesénquima y cubierta muscular esquelética, y cavidad oral pequeña, circunscrita por mandíbula hipoplásica conformada por cartílago. Se revisa la literatura y se reafirma la necesidad de estudio multidisciplinario de esta patología para mejorar su comprensión.

PALABRAS CLAVE: Holoprosencefalia semilobar; Complejo disgnatia; Ciclopía, prosencéfalo; Gastrulación.

\section{INTRODUCCIÓN}

La holoprosencefalia (HPE) se caracteriza por ausencia en la división del prosencéfalo (Raam et al., 2011). Se acompaña de anomalías, que pueden ser faciales y no faciales (Lacbawan et al., 2009; Orioli et al., 2010). Las faciales que se asocian con más frecuencia a casos de HPE son agenesia premaxilar, ciclopía y cebocefalia, y de las no faciales, la transposición de los grandes vasos, polidactilia postaxial, defectos genitales, vertebrales y de reducción de extremidades (Orioli et al., 2010; Winter et al., 2015). La ciclopía generalmente acompaña a los casos graves de HPE (Leoncini et al., 2008; Orioli et al., 2011) y presenta como característica principal una sola órbita o "pseudo órbita" (Orioli et al., 2011). La afectación facial también engloba alteraciones en el desarrollo del primer arco faríngeo, como el encontrado en el complejo holoprosencefalia-agnatia (CHA). Se acompaña de otras malformaciones como: hipoplasia maxilar, implantación baja de pabellones auriculares, sinotia, ausencia de la lengua, microstomía y persistencia de la membrana bucofaríngea (Suárez-Obando \& Prieto, 2007; Kauvar et al., 2010; Oviedo et al., 2011; CoronaRivera et al., 2013).

La HPE es el defecto del prosencéfalo más común, con una prevalencia de 1/10,000 nacimientos (Leoncini et $a l$. .). La ciclopía, asociada a holoprosencefalia tiene una prevalencia de 1/100,000 nacimientos (Orioli et al., 2011). La prevalencia del espectro de agnatia en cohortes de pacientes con HPE es de $0,8 \%$ a $10 \%$ (Kauvar et al.). Actualmente

\footnotetext{
${ }^{1}$ Servicio Social de la Facultad de Medicina y Ciencias biomédicas, Universidad Autónoma de Chihuahua, Chihuahua, Chihuahua, México.

${ }^{2}$ Laboratorio de Embriología de la Facultad de Medicina y Ciencias biomédicas, Universidad Autónoma de Chihuahua, Chihuahua, Chihuahua, México.

${ }^{3}$ Facultad de Odontología, Universidad Autónoma de Chihuahua, Chihuahua, Chihuahua, México.

${ }^{4}$ Anatomopatológo, Universidad Autónoma de Chihuahua, Chihuahua, Chihuahua, México.

Este proyecto fue parcialmente apoyado por la Facultad de Medicina y Ciencias Biomédicas, Universidad Autónoma de Chihuahua, Chihuahua, Chihuahua, México. Proyecto de fortalecimiento de la calidad educativa 2016, clave OP: OP/PFCE-2016-08MSU0017H-05.
} 
existen 144 casos reportados del complejo holoprosencefaliaagnatia, de los cuales, se estima que sólo 32 tienen una descripción adecuada (Gekas et al., 2010; Corona-Rivera et al.; Golinko et al., 2015). El objetivo fue describir las características morfológicas e histopatológicas de un feto humano con holoprosencefalia y sus malformaciones asociadas, así como la revisión de la literatura.

\section{MATERIAL Y MÉTODO}

Previo a su estudio, se solicitó la firma del consentimiento informado. Posteriormente, se realizó estudio morfológico externo e interno, microdisección y estudio histopatológico de un caso de holoprosencefalia semilobar. Las medidas antropométricas se realizaron con un calibrador milimétrico digital Mitutoyo de resolución 0,01mm y cinta métrica flexible de fibra de vidrio. Para llevar a cabo la microdisección se empleó un estereomicroscopio Carl Zeiss* SteREO Discovery.V8 y AxioCam ICc 1 con software AxioVision 4.8, para la captura de imágenes. La microdisección se llevó a cabo con el equipo de cirugía oftálmica como tijeras micro iris Miltex 18-1618, pinzas Peer Vigor Swiss Titanium \#5 y set de hemostatos mosquito.

El estudio histopatológico fue realizado por un experto, quien generó cortes histológicos de 3-5 micras de ojo, cerebro, macizo facial y cavidad oral. Dichos cortes fueron teñidos con hematoxilina y eosina (H-E) para su estudio, mediante microscopía óptica.
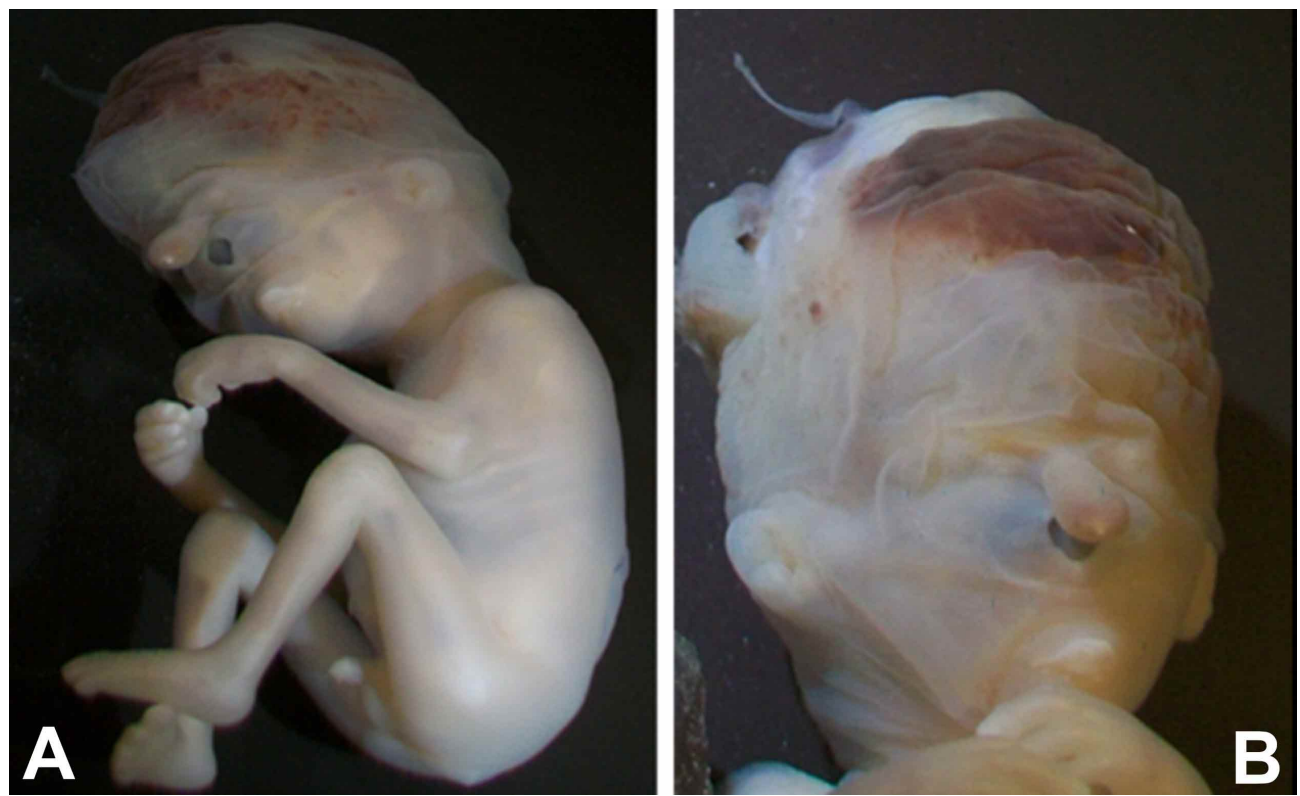

Fig. 1. A. Vista lateral de feto masculino de 12.4-13.2 semanas de gestación, con evidente afectación facial: ciclopía con disgnatia. B. Vista frontal, donde se aprecia esbozo oral hipoplásico sin apertura oral, cubierto por una membrana y sin labios.

\section{RESULTADOS} de $47 \mathrm{~mm}$ y peso de $150 \mathrm{~g}$. por una membrana y sin labios (Fig. 1. B). incompleta del riñón izquierdo.

Descripción clínica. Corresponde a un feto masculino de 12,4-13,2 semanas de gestación, producto de la primera gestación, la edad materna fue de 19 años, sin control prenatal, con etilismo y tabaquismo positivo durante los primeros dos meses de gestación, sin antecedentes personales patológicos de importancia. El reporte sonográfico mostró a un producto con cavidad craneal ocupada por líquido con aparente fusión de los tálamos y nariz en probóscide, resto normal. Se decidió realizar aborto terapéutico por vía vaginal, obteniendo a un feto único con una longitud vértex-cóccix de 71 $\mathrm{mm}$, perímetro cefálico $75 \mathrm{~mm}$, torácico $56 \mathrm{~mm}$, abdominal

Descripción morfológica externa e interna. Los hallazgos morfológicos externos confirman una cavidad orbitaria con globo ocular único en la línea media, por encima de este una probóscide con una longitud de 5,7 mm y un diámetro distal de 2,6 mm, sin fosas nasales (Fig. 1. A). Se aprecia esbozo oral hipoplásico sin apertura oral, cubierto

En la microdisección de cavidades corporales realizada se visualizó todo en posición normal, excepto por la rotación

Estudio histopatológico. El estudio reportó encéfalo con un solo ventrículo y sus hemisferios cerebrales parcialmente separados, bulbos olfatorios ausentes y un nervio óptico 
CERVANTES-FLORES, H. A.; BENCOMO-LOEPPKY, S.; GARCÍA-RIZK, J. A.; LEVARIO-CARRILLO, M.; REZA-LÓPEZ, S. A.; LOYA-LOYA, M. E.; SANÍN, L. H.; FIERROMURGA, R. \& CHÁVEZ-CORRAL, D. V. Holoprosencefalia semilobar y malformaciones asociadas: Reporte de caso y algunas consideraciones. Int. J. Morphol., 37(1):123-127, 2019.

representado por una cintilla delgada. La corteza cerebral, hipófisis, tálamo e hipotálamo con las características histológicas adecuadas para su edad gestacional. Se aprecian a los párpados fusionados, una cavidad orbitaria con su molde cartilaginoso, un solo globo ocular y la conjuntiva y esclerótica normales. Hacia el interior del ojo se identificaron el lente, retina y córnea (Fig. 2. A). En la región facial se encontró probóscide, con cartílago tubular en formación, asociado a mesénquima, y cubierta muscular esquelética. La cavidad oral es pequeña, circunscrita por una mandíbula hipoplásica conformada por cartílago con centros de osificación y mesénquima. En el área oral se apreció la presencia de rabdomioblastos con diferenciación a músculo estriado, conformando la lengua (Fig. 2. B).
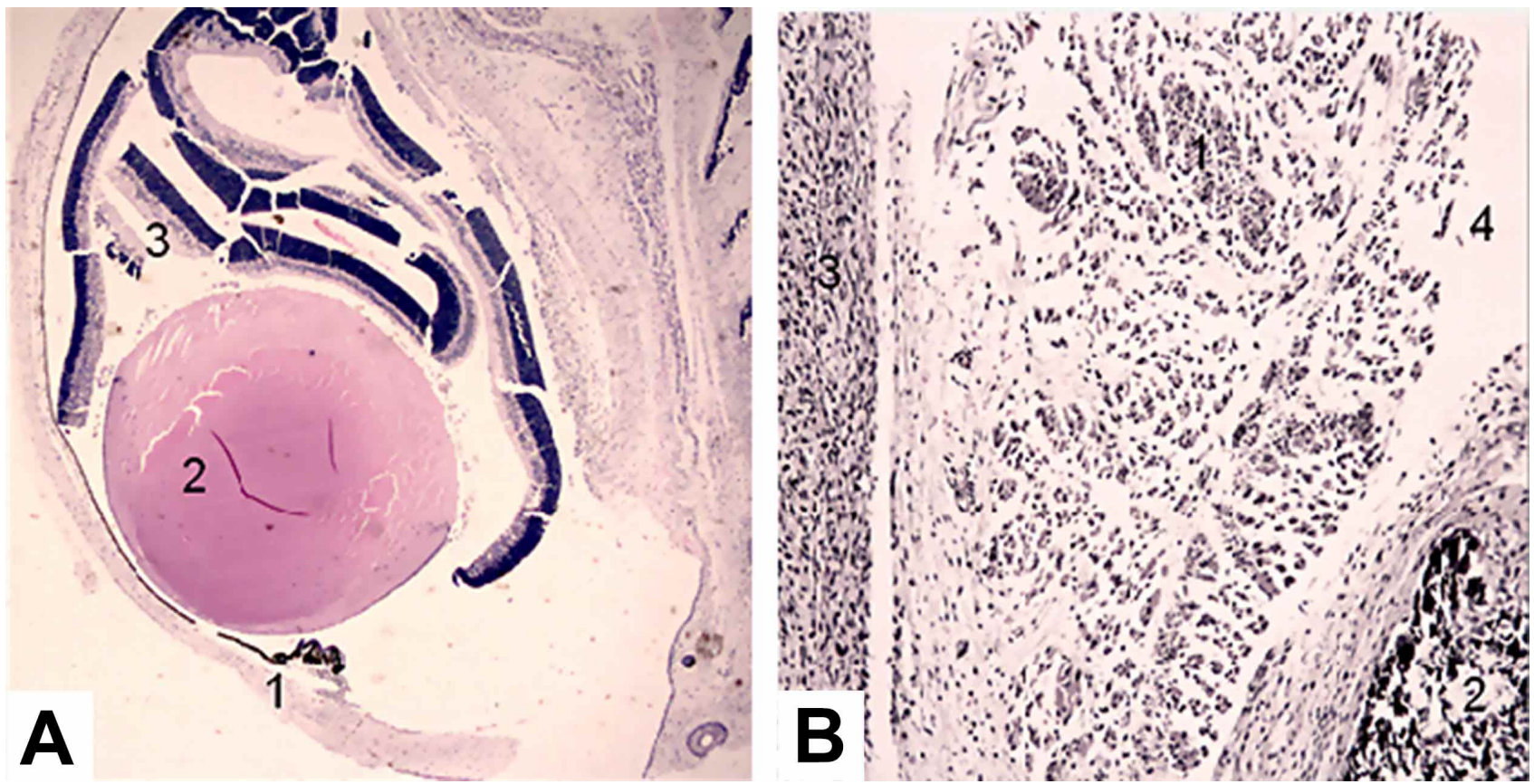

Fig. 2. A. Microfotografía de corte histológico sagital, de globo ocular único, 20x, H-E. Se observa cornea (1), lente (2) y retina (3) únicos. B. Microfotografía de corte histológico transversal de cavidad oral, 20x, H-E. Se aprecia diferenciación de músculo estriado que corresponde a la lengua (1), hueso y cartílago mandibular del lado izquierdo (2), mesénquima (3), pequeña cavidad oral (4).

\section{DISCUSIÓN}

Con los hallazgos descritos este caso corresponde a una HPE semilobar, ciclopía y micrognatia, con ausencia de los labios y apertura oral cubierta por una membrana. La HPE con variante semilobar es la segunda más reportada (9 \%) y de acuerdo con la literatura la ciclopía es la anomalía ocular más frecuente (53\%) en presentación conjunta a la HPE (Kauvar etal.; Corona-Rivera et al.). De acuerdo con la clasificación de Corona Rivera et al., el caso descrito se aproxima al complejo disgnatia con HPE sin sinotia. Sin mencionar de manera explícita anomalías oculares o la presencia de una membrana que cubre la cavidad oral, como son los hallazgos de este estudio.

La HPE semilobar pudo ser provocada por alteraciones en la señalización de los centros telencefálicos, ocurridas durante la $3^{\mathrm{a}}$ a la $5^{\mathrm{a}}$ semanas de gestación (Geng \& Oliver, 2009). El período crítico para que se presente la
HPE es durante la gastrulación, al ocurrir una interrupción durante esta etapa se afecta el desarrollo del prosencéfalo (Shiota \& Yamada, 2010). La placa precordal (PrCP) el endodermo y el tubo neural son importantes para el desarrollo craneofacial, por lo que si ocurre alguna alteración en estas estructuras o una interrupción en sus vías de señalización se manifiesta la variabilidad fenotípica de HPE (Petryk et al., 2015). La simetría prosencefálica está determinada por señales que emite la PrCP, siendo la más representativa el Sonic Hedgehog ( $\mathrm{SHH})$, involucradas en la patogénesis de la HPE (Roessler et al., 2010). La HPE semilobar, en los estadios de Carnegie (CS) 10-14, en modelos murinos, ocurre por una interrupción en los centros de moldeado telencefálicos rostral y ventral, determinando alteraciones del cerebro anterior y del desarrollo de la línea media (Geng \& Oliver; Lacbawan et al.). Asimismo, existe el antecedente de consumo de alcohol de la 
madre durante los primeros dos meses de gestación como un posible factor etiológico (Ronen \& Andrews, 1991) relacionado al deterioro en la expresión de SHH y la apoptosis en las célulasdel PrCP por lo que se altera la formación de la cara, la división del prosencéfalo y la separación del campo óptico primario (Gondré-Lewis et al., 2015; Goswami \& Kusre, 2015).

La formación de los ojos se inicia al final de la gastrulación, donde es necesaria la formación del campo óptico primario y la $\mathrm{PrCP}$ para su división y formación de las placodas ópticas (Roessler et al.). La patogénesis de la ciclopía en modelos murinos involucra la participación de PrCP que regula a SHH para establecer la línea media ventral del cerebro y la subdivisión del campo óptico, y por lo tanto al ocurrir una interrupción en la interrelación de estas resulta la ciclopía (Yamada et al., 2004; Kauvar et al.; Shiota \& Yamada).

La presentación conjunta de las alteraciones craneofaciales se fundamenta en hallazgos encontrados en modelos de embriones murinos deficientes de $\mathrm{SHH}$, donde se ha constatado la presencia de ciclopía y ausencia parcial de huesos craneofaciales. Estos modelos presentan arcos faríngeos con apariencia casi normal al estadio 9.5, y para el 15,5 manifiestan una probóscide, no presentan ojos, nariz, ni estructuras orales fenotípicamente normales. Aunque se considera que tales defectos pueden ser secundarios a defectos del prosencéfalo y del mesencéfalo (Kauvar et al.).

Se ha estimado con base en los resultados de la colección de embriones de Kyoto que ocurre una interferencia molecular o estructural previa al movimiento del campo óptico primario antes de la $5^{\text {a }}$ semana de gestación (Yamada et al.; Shiota \& Yamada).

La embriogénesis de la mandíbula se inicia a finales de la cuarta semana de gestación a partir: del cartílago de Meckel del primer arco faríngeo, de las células de la cresta neural, del endodermo y de la interacción de varias vías moleculares, como SHH. La señalización de SHH secretado por epitelio faríngeo en estadios tempranos, permite la supervivencia de los derivados del mesénquima, la diferenciación y condensación del cartílago del arco mandibular. En embriones murinos con ausencia de SHH el cartílago de Meckel sólo forma un remanente del arco mandibular, sin diferenciarse en las otras estructuras (Melnick et al., 2005; Billmyre \& Klingensmith, 2015).

Se ha constatado una mayor severidad fenotípica de las anomalías craneofaciales cuando existe escasa cantidad del mesénquima que circunda al prosencéfalo y al perteneciente al proceso frontonasal (Shiota \& Yamada).

\section{CONCLUSIONES}

La recomendación ante la presencia de casos con holoprosencefalia, es su estudio por un grupo especializado (morfólogos, genetistas, pediatras, neurólogos y epidemiólogos) ya que esto permitirá entender su desarrollo y se podrá buscar su asociación con factores de riesgo, lo cual resalta la importancia de recabar la información a través de la historia clínica completa y de los antecedentes heredofamiliares. La HPE, acompañada de ciclopía y malformaciones de la mandíbula comparten algunos aspectos dentro de la embriogénesis, fisiopatología y los factores de riesgo lo cual determina su fenotipo y severidad, sin embargo las anomalías del esbozo oral y la membrana descritos en el caso no fueron corroborados en la literatura revisada.

CERVANTES-FLORES, H. A.; BENCOMO-LOEPPKY, S.; GARCÍA-RIZK, J. A.; LEVARIO-CARRILLO, M.; REZALÓPEZ, S. A.; LOYA-LOYA, M. E.; SANÍN, L. H.; FIERROMURGA, R. \& CHÁVEZ-CORRAL, D. V. Semilobar holoprosencephaly and associated malformations: Case report and some considerations. Int. J. Morphol., 37(1):123-127, 2019.

SUMMARY: The most common defect of the forebrain is holoprosencephaly (HPE), characterized by absence in the forebrain division. Holoprosencephaly has a prevalence of $1 / 10,000$ in newborns; the cyclopia of $1 / 100,000$ births and the agnathia, in a series of cases of holoprosencephaly ranges from 0.8 to $10 \%$. The objective was the description of the morphological and histopathological characteristics of fetus with holoprosencephaly and its associated malformations. A male fetus was studied. Microdissection was performed under the stereomicroscope, taking microphotographs with AxioCam camera and AxioVision 4.8 software, and histopathological study. The estimated gestational age was 12.4-13.2 weeks, the findings were semilobar HPE, associated with cyclopia, hypoplastic oral outline without buccal opening, covered by a membrane and lips absence. The histopathological study reported: eye with lens, retina and cornea only; in the face, proboscis with tubular cartilage in formation associated with mesenchyme and musculoskeletal sheath, and small oral cavity, delimited by hypoplastic mandible conformed by cartilage. The literature is reviewed and reaffirmed the need for multidisciplinary studies of this disease to improve their understanding.

KEY WORDS: Semilobar holoprosencephaly; Dysgnathia complex; Cyclopia, prosencephalon; Gastrulation.

\section{REFERENCIAS BIBLIOGRÁFICAS}

Billmyre, K. K. \& Klingensmith, J. Sonic hedgehog from pharyngeal arch 1 epithelium is necessary for early mandibular arch cell survival and later cartilage condensation differentiation. Dev. Dyn., 244(4):564-76, 2015. 
Corona-Rivera, J. R.; Trujillo-Ponce, S. A.; Barrios-Prieto, E.; QuilesCorona, M.; Miguel-Jimenez, K.; Aguirre-Guillen, R. L.; BobadillaMorales, L. \& Corona-Rivera, A. Dysgnathia complex sine holoprosencephaly nor synotia: a case report and discussion of its nosology. Genet. Couns., 24(1):45-55, 2013.

Gekas, J.; Li, B. \& Kamnasaran, D. Current perspectives on the etiology of agnathia-otocephaly. Eur. J. Med. Genet., 53(6):358-66, 2010.

Geng, X. \& Oliver, G. Pathogenesis of holoprosencephaly. J. Clin. Invest., 119(6):1403-13, 2009.

Golinko, M. S.; Shetye, P.; Flores, R. L. \& Staffenberg, D. A. Severe agnathia-otocephaly complex: Surgical management and longitudinal follow-up from birth through adulthood. J. Craniofac. Surg., 26(8):2387-92, 2015.

Gondré-Lewis, M. C.; Gboluaje, T.; Reid, S. N.; Lin, S.; Wang, P.; Green, W.; Diogo, R.; Fidélia-Lambert, M. N. \& Herman, M. M. The human brain and face: mechanisms of cranial, neurological and facial development revealed through malformations of holoprosencephaly, cyclopia and aberrations in chromosome 18. J. Anat., 227(3):25567, 2015

Goswami, D. \& Kusre, G. Agnathia holoprosencephaly and situs inversus in a neonate born to an alcoholic mother. J. Clin. Diagn. Res., 9(5):AD01-2, 2015.

Kauvar, E. F.; Solomon, B. D.; Curry, C. J.; van Essen, A. J.; Janssen, N.; Dutra, A.; Roessler, E. \& Muenke, M. Holoprosencephaly and agnathia spectrum: Presentation of two new patients and review of the literature. Am. J. Med. Genet. C Semin. Med. Genet., 154C(1):15869, 2010.

Lacbawan, F.; Solomon, B. D.; Roessler, E.; El-Jaick, K.; Domené, S.; Velez, J. I.; Zhou, N.; Hadley, D.; Balog, J. Z.; Long, R.; Fryer, A.; Smith, W.; Omar, S.; McLean, S. D.; Clarkson, K.; Lichty, A.; Clegg, N. J.; Delgado, M. R.; Levey, E.; Stashinko, E.; Potocki, L.; Vanallen, M. I.; Clayton-Smith, J.; Donnai, D.; Bianchi, D. W.; Juliusson, P. B.; Njølstad, P. R.; Brunner, H. G.; Carey, J. C.; Hehr, U.; Müsebeck, J.; Wieacker, P. F.; Postra, A.; Hennekam, R. C.; van den Boogaard, M. J.; van Haeringen, A.; Paulussen, A.; Herbergs, J.; SchranderStumpel, C. T.; Janecke, A. R.; Chitayat, D.; Hahn, J.; McDonaldMcGinn, D. M.; Zackai, E. H.; Dobyns, W. B. \& Muenke, M. Clinical spectrum of SIX3-associated mutations in holoprosencephaly: correlation between genotype, phenotype and function. J. Med. Genet., 46(6):389-98, 2009.

Leoncini, E.; Baranello, G.; Orioli, I. M.; Annerén, G.; Bakker, M.; Bianchi, F.; Bower, C.; Canfield, M. A.; Castilla, E. E.; Cocchi, G.; Correa, A.; De Vigan, C.; Doray, B.; Feldkamp, M. L.; Gatt, M.; Irgens, L. M.; Lowry, R. B.; Maraschini, A.; Mc Donnell, R.; Morgan, M.; Mutchinick, O.; Poetzsh, S.; Riley, M.; Ritvanen, A.; Gnansia, E. R.; Scarano, G.; Sipek, A.; Tenconi, R. \& Mastroiacovo, P. Frequency of holoprosencephaly in the International Clearinghouse Birth Defects Surveillance Systems: searching for population variations. Birth Defects Res. A Clin. Mol. Teratol., 82(8):585-91, 2008.

Melnick, M.; Witcher, D.; Bringas, P. Jr.; Carlsson, P. \& Jaskoll, T. Meckel's cartilage differentiation is dependent on hedgehog signaling. Cells Tissues Organs, 179(4):146-57, 2005.

Orioli, I. M. \& Castilla, E. E. Epidemiology of holoprosencephaly: Prevalence and risk factors. Am. J. Med. Genet. C Semin. Med. Genet., 154C(1):13-21, 2010.

Orioli, I. M.; Amar, E.; Bakker, M. K.; Bermejo-Sánchez, E.; Bianchi, F.; Canfield, M. A.; Clementi, M.; Correa, A.; Csáky-Szunyogh, M.; Feldkamp, M. L.; Landau, D.; Leoncini, E.; Li, Z.; Lowry, R. B.; Mastroiacovo, P.; Morgan, M.; Mutchinick, O. M.; Rissmann, A.; Ritvanen, A.; Scarano, G.; Szabova, E. \& Castilla, E. E. Cyclopia: an epidemiologic study in a large dataset from the International Clearinghouse of Birth Defects Surveillance and Research. Am. J. Med. Genet. C Semin. Med. Genet., 157(4):344-57, 2011.

Oviedo, P.; Ruoti Cosp, M.; Mendoza, L.; Ontano, M. \& Irala, L. Diagnóstico prenatal del complejo agnatia holoprosencefalia. Mem. Inst. Investig. Cienc. Salud, 9(1):54-9, 2011.
Petryk, A.; Graf, D. \& Marcucio, R. Holoprosencephaly: signaling interactions between the brain and the face, the environment and the genes, and the phenotypic variability in animal models and humans. Wiley Interdiscip. Rev. Dev. Biol., 4(1):17-32, 2015.

Raam, M. S.; Solomon, B. D. \& Muenke, M. Holoprosencephaly: a guide to diagnosis and clinical management. Indian Pediatr., 48(6):457-66, 2011.

Roessler, E. \& Muenke, M. The molecular genetics of holoprosencephaly. Am. J. Med. Genet. C Semin. Med. Genet., 154C(1):52-61, 2010.

Ronen, G. M. \& Andrews, W. L. Holoprosencephaly as a possible embryonic alcohol effect. Am. J. Med. Genet., 40(2):151-4, 1991.

Shiota, K. \& Yamada, S. Early pathogenesis of holoprosencephaly. Am. J. Med. Genet. C. Semin. Med. Genet., 154C(1):22-8, 2010.

Suárez-Obando, F. \& Prieto, J. C. Complejo agnatia holoprosencefalia: informe de caso. Colomb. Med., 38(3):305-7, 2007.

Winter, T. C.; Kennedy, A. M. \& Woodward, P. J. Holoprosencephaly: a survey of the entity, with embryology and fetal imaging. Radiographics, 35(1):275-90, 2015.

Yamada, S.; Uwabe, C.; Fujii, S. \& Shiota, K. Phenotypic variability in human embryonic holoprosencephaly in the Kyoto Collection. Birth Defects Res. A Clin. Mol. Teratol., 70(8):495-508, 2004.

\author{
Dirección para correspondencia: \\ Dora Virginia Chávez Corral \\ Laboratorio de Embriología \\ Facultad de Medicina y Ciencias Biomédicas \\ Circuito Universitario Campus II \\ Universidad Autónoma de Chihuahua \\ Chihuahua \\ MÉXICO
}

\author{
Email: dochavez@uach.mx \\ dcorral@prodigy.net.mx
}

Recibido :16-07-2018

Aceptado: 27-11-2018 